\title{
Modelling of Lock-in Infrared Thermography for Delamination Inspection in Glass Fiber-Reinforced Composites
}

\author{
Ranjit Shrestha ${ }^{1}$, Yoonjae Chung ${ }^{1}$ and Wontae $\mathrm{Kim}^{2 \dagger}$ \\ ${ }^{1}$ Department of Mechanical Engineering, Kongju National University, 1223-24 Cheonan- \\ daero, Seobuk-gu, Cheonan-si, Chungcheongnam-do, 31080, South Korea \\ ${ }^{2}$ Devi. of Mechanical \& Automotive Engineering, Kongju National University, 1223-24 \\ Cheonan-daero, Seobuk-gu, Cheonan-si, Chungcheongnam-do, 31080, South Korea \\ ${ }^{\dagger}$ Corresponding author. E-mail address: kwt@kongju.ac.kr
}

\begin{abstract}
:
The increasing use of composite materials in various industries has evidenced the need for development of more effective nondestructive evaluation methodologies to reduce rejected parts and optimized the production cost. In this study, a transient thermal finite element model has been created and a sinusoidal thermal source at different excitation frequency was used to excite the test sample following the lock-in infrared thermography experimental procedure. The influence of applied heat flux and excitation frequency on various shape, size and depth of delamination is analyzed to investigate the thermal response. The thermal data processing with Fourier transform was performed to reap the phase angle information and the relationship with delamination shape, size and depth was developed. The model enables a better understanding of lock-in infrared thermography and provides a means of inspection of glass fiber-reinforced composites.
\end{abstract}

